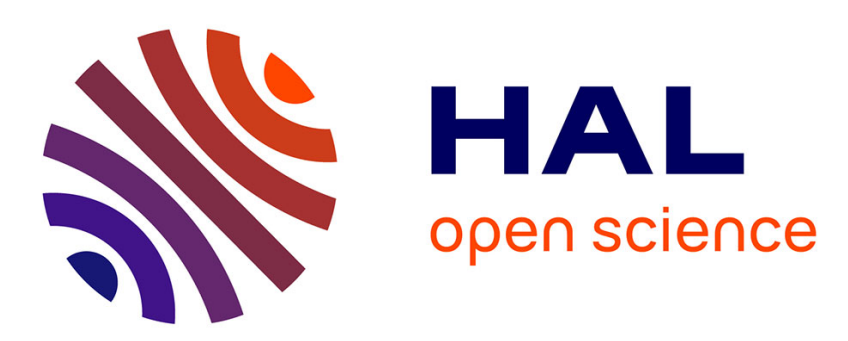

\title{
A method to compute the transition function of a piecewise deterministic Markov process with application to reliability
}

Julien Chiquet, Nikolaos Limnios

\section{- To cite this version:}

Julien Chiquet, Nikolaos Limnios. A method to compute the transition function of a piecewise deterministic Markov process with application to reliability. Statistics and Probability Letters, 2010, 78 (12), pp.1397. 10.1016/j.spl.2007.12.016 . hal-00634353

\section{HAL Id: hal-00634353 \\ https://hal.science/hal-00634353}

Submitted on 21 Oct 2011

HAL is a multi-disciplinary open access archive for the deposit and dissemination of scientific research documents, whether they are published or not. The documents may come from teaching and research institutions in France or abroad, or from public or private research centers.
L'archive ouverte pluridisciplinaire HAL, est destinée au dépôt et à la diffusion de documents scientifiques de niveau recherche, publiés ou non, émanant des établissements d'enseignement et de recherche français ou étrangers, des laboratoires publics ou privés. 


\section{Accepted Manuscript}

A method to compute the transition function of a piecewise

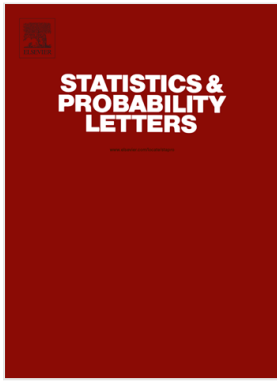

PII:

DOI:

10.1016/j.spl.2007.12.016

Reference: $\quad$ STAPRO 4853

To appear in: $\quad$ Statistics and Probability Letters

Received date: 5 January 2007

Revised date: 19 September 2007

Accepted date: 11 December 2007

Please cite this article as: Chiquet, J., Limnios, N., A method to compute the transition function of a piecewise deterministic Markov process with application to reliability. Statistics and Probability Letters (2007), doi:10.1016/j.spl.2007.12.016

This is a PDF file of an unedited manuscript that has been accepted for publication. As a service to our customers we are providing this early version of the manuscript. The manuscript will undergo copyediting, typesetting, and review of the resulting proof before it is published in its final form. Please note that during the production process errors may be discovered which could affect the content, and all legal disclaimers that apply to the journal pertain. 


\title{
A Method to Compute the Transition Function of a Piecewise Deterministic Markov Process with Application to Reliability
}

\author{
Julien Chiquet ${ }^{\mathrm{a}, *}{ }^{*}$ Nikolaos Limnios ${ }^{\mathrm{a}}$ \\ ${ }^{a}$ Laboratoire de Mathématiques Appliquées de Compiègne, \\ Université de Technologie de Compiègne, France
}

\begin{abstract}
We study the time evolution of an increasing stochastic process governed by a first order stochastic differential system. This defines a particular piecewise deterministic Markov process (PDMP). We consider a Markov renewal process (MRP) associated to the PDMP and its Markov renewal equation (MRE) which is solved in order to obtain a closed-form solution of the transition function of the PDMP. It is then applied in the framework of survival analysis to evaluate the reliability function of a given system. We give a numerical illustration and we compare this analytical solution with the Monte-Carlo estimator.
\end{abstract}

Key words: Piecewise deterministic Markov Process, Markov Renewal Process, Transition function, Reliability, Survival Analysis

\footnotetext{
* Corresponding author

Email addresses: julien.chiquet@utc.fr (Julien Chiquet), nikolaos.limnios@utc.fr (Nikolaos Limnios).
} 


\section{Introduction}

In the literature of stochastic differential systems, a large part is dedicated to those involving diffusion type processes, that lead to the theory of stochastic differential equations (see, e.g., Øksendal, 2003). Another class of stochastic models has arisen to describe the random evolution of processes that do not involve diffusion type motion, but rather the mixture of deterministic motions and random jumps. Such an idea has been suggested by various authors, yet Davis provided a major contribution to this approach : he gave the underlying theory for the class of stochastic models called piecewise deterministic Markov processes (PDMP) in Davis (1984), farther developed in Davis (1993) and Jacobsen (2006). In Koroliuk and Limnios (2005) the limit theory is studied, in a functional setting. Lapeyre and Pardoux (2003) worked with transport processes, which are a special case of PDMP, in order to give a stochastic interpretation of the transport equations used in physics for the modeling of the motion of particles. This family of processes are nowadays much used in several applications, e.g., reliability analysis (Devooght and Smidts, 1996) or insurance (Embrechts et al., 2001). In Chiquet and Limnios (2006); Chiquet et al. (to appear), we took advantage of a PDMP for the modeling of degradation mechanisms arising in a structure subject to random environmental effects.

The main contribution of this paper is to provide a closed-form solution for the transition function of a PDMP defined through a first order differential equation. For this purpose, we associate to this PDMP a Markov renewal process (MRP), thus we will refer to results from this theory (see e.g. Limnios and Opriçan, 2001).

The outline is the following: in section 2, we give the model settings and standard notation for MRP. We then build and solve a Markov renewal equation (MRE) for the transition function of the PDMP. In section 3, an application for survival analysis is studied, where the numerical computation of the reliability is detailed. We compare the result obtained through the direct resolution of the MRE with the empirical estimator obtained with the Monte-Carlo method. 


\section{Transition function of the PDMP}

Let $\left(Z_{t}, t \in \mathbb{R}_{+}\right)$be a real-valued stochastic process starting almost surely from $z>0$ and governed by the following first order differential system

$$
\dot{Z}_{t}=C\left(Z_{t}, X_{t}\right), \quad Z_{0}=z,
$$

with the following assumptions:

A. 1 The process $\left(X_{t}, t \in \mathbb{R}_{+}\right)$is an irreducible Markov process with a countable state space $E$, an initial distribution $\alpha_{i}=\mathbb{P}\left(X_{0}=i\right)$, and a matrix generator $\mathbf{A}=\left(a_{i j}\right)_{i, j \in E}$ such that $a_{i j} \geq 0$, for all $i \neq j$, and $a_{i i}=-a_{i}=-\sum_{k \in E, i \neq k} a_{i k}$.

A. 2 The function $C: \mathbb{R}_{+} \times E \longrightarrow \mathbb{R}_{+}$is measurable, strictly positive and Lipschitz w.r.t. the first argument, i.e, there is a function $f: E \longrightarrow \mathbb{R}$, for $x, y \in \mathbb{R}_{+}$and $i \in E$, such that $|C(x, i)-C(y, i)| \leq f(i)|x-y|$.

Each path $Z_{t}(\omega)$ is built in a piecewise manner according to the function $C$ and a path $X_{t}(\omega)$ of the jump Markov process. That is, for $t \in \mathbb{R}_{+}$, the Cauchy problem

$$
\dot{Z}_{t}(\omega)=C\left(Z_{t}(\omega), i\right), \quad Z_{0}(\omega)=z, \quad i \in E,
$$

has a unique solution built on the successive intervals $\left[S_{n}(\omega), S_{n+1}(\omega)\right)$, where $\left(S_{n}, n \in \mathbb{N}\right)$ is a random sequence describing the jump times of $X_{t}$. For any $t<$ $S_{1}(\omega)$, we denote by $\varphi_{z, i}(t)$ the solution of $(2)$, where $X_{0}(\omega)=i$, i.e., $\varphi_{z, i}(t)$ is the solution before the first jump time of $X_{t}$, conditionally to the starting value of $\left(Z_{0}, X_{0}\right)=(z, i)$. We also assume that $Z_{0}$ and $X_{0}$ are independent. In the sequel, we focus on the transition function $P$, defined by

$$
P_{i j}(z, B, t):=\mathbb{P}_{z, i}\left(Z_{t} \in B, X_{t}=j\right), \quad i, j \in E, B \in \mathcal{B},
$$

where $B$ is a subset of $\mathcal{B}$, the Borel $\sigma$-field of $\mathbb{R}_{+}$and $\mathbb{P}_{z, i}(\cdot):=\mathbb{P}\left(\cdot \mid Z_{0}=z, X_{0}=i\right)$.

A Markov process is a special MRP, thus we may associate to $\left(Z_{t}, X_{t}\right)$ the extended $\operatorname{MRP}\left(\zeta_{n}, Y_{n}, S_{n}, n \in \mathbb{N}\right)$ such as

$$
\zeta_{n}=Z_{S_{n}}, \quad Y_{n}=X_{S_{n}}, \quad n \in \mathbb{N} .
$$


The process $\left(Y_{n}, S_{n}\right)$ is a standard MRP, while $\left(\zeta_{n}, Y_{n}, S_{n}\right)$ is an extended one. In the homogeneous case the associated semi-Markov kernel $Q$ is defined, for $t>0$, by

$$
Q_{i j}(z, B, t):=\mathbb{P}_{z, i}\left(\zeta_{1} \in B, Y_{1}=j, S_{1}-S_{0} \leq t\right)
$$

The Stieltjes-convolution is denoted by “*”, hence, the convolution of $Q$ with a measurable function $\phi$ on the space $\mathbb{R}_{+} \times E$ is

$$
(Q * \phi)_{i j}(z, B, t)=\sum_{k \in E} \int_{\mathbb{R}_{+}} \int_{0}^{t} Q_{i k}(z, \mathrm{~d} y, \mathrm{~d} s) \phi_{k j}(y, B, t-s),
$$

for $i, j \in E, z>0$ and $B \in \mathcal{B}$. In the same way, the successive $n$-fold convolutions of the semi-Markov kernel are defined recursively. For $n=0,1$,

$$
Q_{i j}^{(0)}(z, B, t)=\mathbb{1}_{\{i=j\}} \mathbb{1}_{B}(z) \mathbb{1}_{\mathbb{R}_{+}}(t), \quad Q_{i j}^{(1)}(z, B, t)=Q_{i j}(z, B, t),
$$

where $\mathbb{1}_{B}(x)$ is the indicator function, i.e., $\mathbb{1}_{B}(x)=1$ if $x \in B, 0$ otherwise. For $n \geq 2$, the $n$-fold convolution turns to

$$
\begin{aligned}
Q_{i j}^{(n)}(z, B, t) & :=\left(Q * Q^{(n-1)}\right)_{i j}(z, B, t) \\
& =\sum_{k \in E} \int_{\mathbb{R}_{+}} \int_{0}^{t} Q_{i k}(z, \mathrm{~d} y, \mathrm{~d} s) Q_{k j}^{(n-1)}(y, B, t-s) .
\end{aligned}
$$

The Markov renewal function $\Psi$, which plays a central role, is defined by

$$
\Psi_{i j}(z, B, t)=\sum_{n \geq 0} Q_{i j}^{(n)}(z, B, t) .
$$

In the case at hand, we have $\left(\zeta_{n}, Y_{n}, S_{n}\right)$ a normal MRP, that is, $\Psi_{i j}(z, B, t)<\infty$ for any fixed $t>0, z>0, B \in \mathcal{B}$ and $i, j \in E$.

A MRE has the following form

$$
\Theta_{i j}(z, B, t)=g_{i j}(z, B, t)+(Q * \Theta)_{i j}(z, B, t),
$$

where $g$ is a known function defined on $\mathbb{R}_{+} \times E \times \mathbb{R}_{+}$and $\Theta$ is the unknown function. The solution (see e.g Koroliuk and Limnios, 2005) is given by

$$
\Theta_{i j}(z, B, t)=(\Psi * g)_{i j}(z, B, t) .
$$

Let us now take advantage of Markov renewal theory to build a solvable MRE for $P$. For this purpose, we first need to calculate $Q$. This is done in the following Lemma. 
Lemma 1 The semi-Markov kernel $Q$ of the $\operatorname{MRP}\left(\zeta_{n}, Y_{n}, S_{n}\right)$ verifies, for $i \neq j$,

$$
Q_{i j}(z, B, \mathrm{~d} t)=a_{i j} e^{-a_{i} t} \delta_{\varphi_{z, i}(t)}(B) \mathrm{d} t
$$

where $\delta_{x}(B)$ is the Dirac distribution, equal to 1 if $x \in B, 0$ otherwise.

PROOF. Assuming that $S_{0}=0$, and conditioning on definition (4), we get

$$
Q_{i j}(z, B, \mathrm{~d} t)=\mathbb{P}_{z, i}\left(Y_{1}=j, S_{1} \in \mathrm{d} t\right) \mathbb{P}_{z, i}\left(\zeta_{1} \in B \mid Y_{1}=j, S_{1}=t\right)
$$

First, since $S_{1}$ and $Y_{1}$ are independent, we have $\mathbb{P}_{z, i}\left(Y_{1}=j, S_{1} \in \mathrm{d} t\right)=a_{i j} e^{-a_{i} t} \mathrm{~d} t$ from usual results of Markov theory. Second, $Z_{t}$ is fully characterized by $\varphi_{z, i}(t)$ before the first jump time $S_{1}$, thus $\mathbb{P}_{z, i}\left(\zeta_{1} \in B \mid X_{1}=j, S_{1}=t\right)=\mathbb{P}_{z, i}\left(Z_{t} \in B\right)=$ $\delta_{\varphi_{z, i}(t)}(B)$. Indeed, the probability $\mathbb{P}_{z, i}\left(Z_{t} \in B\right)$ is zero everywhere, excepted for the time points where $B$ is reached. We hence get the expected result.

Proposition 2 The transition function $P$ of $\left(Z_{t}, X_{t}\right)$ is governed by the MRE

$$
P_{i j}(z, B, t)=g_{i j}(z, B, t)+(Q * P)_{i j}(z, B, t),
$$

whose solution is $P_{i j}(z, B, t)=(\Psi * g)_{i j}(z, B, t)$ and where

$$
g_{i j}(z, B, t)=e^{-a_{i} t} \mathbb{1}_{\{i=j\}} \mathbb{1}_{B}\left(\varphi_{z, i}(t)\right) .
$$

PROOF. It is convenient to make appear $S_{1}$ in (3). Hence,

$$
P_{i j}(z, B, t)=\underbrace{\mathbb{P}_{z, i}\left(Z_{t} \in B, X_{t}=j, S_{1}>t\right)}_{P_{1}}+\underbrace{\mathbb{P}_{z, i}\left(Z_{t} \in B, X_{t}=j, S_{1} \leq t\right)}_{P_{2}} .
$$

Before the first jump of $X_{t}, i=j$ and $Z_{t}$ evolves according to $\varphi_{z, i}(t)$. Thus, $P_{1}=$ $e^{-a(i) t} \mathbb{1}_{B}\left(\varphi_{t}(z, i)\right) \mathbb{1}_{\{i=j\}}$. From Total Probability Theorem, it holds for $P_{2}$ that

$$
P_{2}=\sum_{\substack{k \in E \\ k \neq i}} \int_{0}^{t} \mathbb{P}_{z, i}\left(Z_{t} \in B, X_{t}=j \mid Y_{1}=k, S_{1}=s\right) \mathbb{P}_{z, i}\left(Y_{1}=k, S_{1} \in \mathrm{d} s\right) .
$$

As long as $\mathbb{P}_{z, i}\left(Y_{1}=k, S_{1} \in \mathrm{d} s\right)=a_{i k} e^{-a_{i} s} \mathrm{~d} s$, and noticing that $\mathbb{P}_{z, i}\left(Z_{t} \in B, X_{t}=\right.$ $\left.j \mid Y_{1}=k, S_{1}=s\right)=P_{k j}\left(\varphi_{z, i}(s), B, t-s\right)$, then $P_{2}$ is fully characterized. Finally,

$$
P_{i j}(z, B, t)=e^{-a_{i} t} \mathbb{1}_{\{i=j\}} \mathbb{1}_{B}\left(\varphi_{z, i}(t)\right)+\sum_{\substack{k \in E \\ k \neq i}} a_{i k} \int_{0}^{t} e^{-a(i) s} P_{k j}\left(\varphi_{z, i}(s), B, t-s\right) \mathrm{d} s,
$$


which may be written, with $Q$ given by (9) and $g$ given by (10), as

$$
P_{i j}(z, B, t)=g_{i j}(z, B, t)+\sum_{k \in E} \int_{\mathbb{R}_{+}} \int_{0}^{t} Q_{i k}(z, \mathrm{~d} y, \mathrm{~d} s) P_{k j}(y, B, t-s)
$$

This last equation is of the general form (7), whose solution is given by (8).

\section{Application to reliability}

As an application, let us now study system (1) under assumptions A.1, A.2 and $E$ a finite state space. The system remains reliable until $Z_{t}$ reaches a critical threshold $\Delta>z>0$ at random time $\tau$, which is the failure time, with a cumulative distribution function $F_{\tau}$. A schematic view of the parallel evolution of both components $Z_{t}$ and $X_{t}$ can be seen on Figure 1, where three typical paths of the PDMP are plotted. Of course, since, $C>0, Z_{t}$ is an increasing process. Looking towards reliability

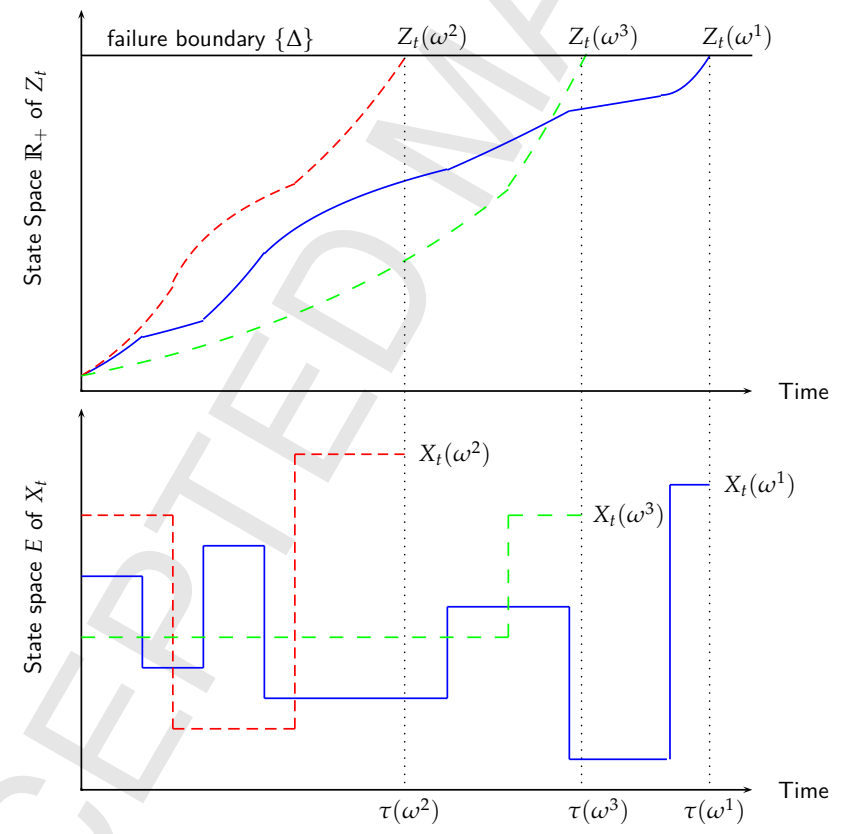

Fig. 1. Schematic view of the PDMP - three paths of $\left(Z_{t}, X_{t}\right)$

analysis, we put $U=[z, \Delta)$ and $D=[\Delta, \infty)$ the respective sets of good states and failure states for $Z_{t}$. Due to the continuous, increasing evolution of $Z_{t}$, it necessarily 
passes through $\Delta$. The reliability function $R$ is defined as follows:

$$
R(t)=\mathbb{P}\left(\left(Z_{t}, X_{t}\right) \in U \times E\right)=\sum_{i, j \in E} \alpha_{i} P_{i j}(z, U, t)=1-F_{\tau}(t) .
$$

Through Proposition 2, $P$ is known. Hence $R$ (as well as $F_{\tau}$ ) is fully characterized:

$$
R(t)=\sum_{i, j \in E} \alpha_{i} \times(\Psi * g)_{i j}(z, U, t)
$$

Note that the kernel $Q$ can be calculated at a given time point $t>0$ for the Borel subset $U$, by integrating (9). As a matter of fact, it holds that

$$
Q_{i j}(z, U, t)=a_{i j} \int_{0}^{t} e^{-a_{i} s} \mathbb{1}_{U}\left(\varphi_{z, i}(s)\right) \mathrm{d} s=\frac{a_{i j}}{a_{i}}\left(1-e^{-a_{i} \min \left(t_{z, i}(\Delta), t\right)}\right),
$$

where $t_{z, i}(\Delta)=\inf \left\{t \geq 0: \varphi_{z, i}(t) \geq \Delta\right\}$.

\subsection{Numerical implementation}

The numerical calculation of $R$ successively requires the kernel $Q$, the $n$-fold convolutions $Q^{(n)}$ for each $n \geq 0$, the Markov renewal function $\Psi$ built upon the $Q^{(n)}$ and the transition function $P$, by a convolution between $g$ and $\Psi$. Since convolution products are time consuming, any simplification would mean a great time saving. By (9), the $n$-fold convolution (5) of $Q$ turns to

$$
Q_{i j}^{(n)}(z, B, t)=\sum_{\substack{k \in E \\ k \neq i}} a_{i k} \int_{0}^{t} e^{-a_{i} s} Q_{k j}^{(n-1)}\left(\varphi_{z, i}(s), B, t-s\right) \mathrm{d} s,
$$

hence removing the integral on $\mathbb{R}_{+}$, thanks to the Dirac distribution. Since our main interest is the reliability, we compute $P$ just for the subset $B \equiv U$, that is,

$$
P_{i j}(z, U, t)=\int_{U} \int_{0}^{t} \Psi_{i j}(z, \mathrm{~d} y, \mathrm{~d} s) e^{-a_{j}(t-s)} \mathbb{1}_{U}\left(\varphi_{y, j}(t-s)\right) .
$$

Indeed, the sum on $E$ has been removed thanks to the structure of $g$. Furthermore, the integration on $y \in \mathbb{R}_{+}$is limited on $U$ since $\mathbb{1}_{U}\left(\varphi_{y, j}(t-s)\right)$ is zero elsewhere.

Theses functions have to be properly discretized to achieve the numerical computation. In the following, a function with an upper-index "\#" means its discretized 
version. This discretization must be operated on both intervals $U=[z, \Delta)$ and $[0, t]$, thus we set two numerical partitions $\left\{z=y_{0}<y_{1}<\cdots<y_{\ell}<\cdots<y_{L}=\Delta^{-}\right\}$and $\left\{0=t_{0}<t_{1}<\cdots<t_{m}<\cdots<t_{M}=t\right\}$. Both $L$ and $M$, being the respective numbers of discretization steps for $[z, \Delta)$ and $[0, t]$, have to be sufficiently large. When $L, M \rightarrow \infty$ each numerical function tends to the associated "true" one. For instance, when $L, M \rightarrow \infty$, then $Q^{\#} \rightarrow Q$ uniformly w.r.t a given matrix norm, by example, $\|Q\|=\max _{i, j} Q_{i j}(z, y, t)$ with $t, z, B$ fixed. Hence, the numerical version of (13) is

$$
P_{i j}^{\#}(z, U, t)=\sum_{y_{\ell} \in[z, \Delta)} \sum_{t_{m} \in(0, t]} \Delta_{y t} \Psi_{i j}^{\#}\left(z, y_{\ell}, t_{m}\right) e^{-a_{j}\left(t-t_{m}\right)} \mathbb{1}_{\varphi_{y_{\ell}, j}\left(t-t_{m}\right)}(U),
$$

where $\Delta_{y t} \Psi_{i j}^{\#}\left(z, y_{\ell}, t_{m}\right)$ is the only unknown, which stands for the numerical evaluation of $\Psi(z, \mathrm{~d} y, \mathrm{~d} s)$ in (13). By the definition (6), it can be evaluated through

$$
\Delta_{y t} \Psi_{i j}^{\#}\left(z, y_{\ell}, t_{m}\right)=\sum_{n \geq 0} \Delta_{y t} Q^{\#(n)}\left(z, y_{\ell}, t_{m}\right)
$$

The derivative $\Delta_{y t} Q^{\#(n)}$ is estimated by finite differences on $y$ and $t$ :

$$
\begin{aligned}
\Delta_{y t} Q^{\#(n)}\left(z, y_{\ell}, t_{m}\right)=\left[Q^{\#(n)}\left(z, y_{\ell}, t_{m}\right)-Q^{\#(n)}\left(z, y_{\ell-1}, t_{m}\right)\right] & \\
- & {\left[Q^{\#(n)}\left(z, y_{\ell}, t_{m-1}\right)-Q^{\#(n)}\left(z, y_{\ell-1}, t_{m-1}\right)\right] . }
\end{aligned}
$$

Each element $Q^{\#(n)}$ in (15) is obtained by the numerical version of (12):

$$
Q_{i j}^{\#(n)}\left(z, y_{\ell}, t_{m}\right)=\sum_{\substack{k \in E \\ k \neq i}} a_{i k} \sum_{t_{m} \in(0, t]} e^{-a_{i} t_{m}} Q_{k j}^{\#(n-1)}\left(\varphi_{z, i}\left(t_{m}\right), y_{\ell}, t-t_{m}\right) \Delta t_{m},
$$

with $\Delta t_{m}=t_{m}-t_{m-1}$, the time-step discretization. Finally, we point out that the sum on the $n$-fold convolutions of the kernel in (14) is truncated from the rank $n^{*}$, provided that $\left\|Q^{\#\left(n^{*}\right)}\right\|<\varepsilon$. We put $\varepsilon$ a small real number, chosen closed to the machine precision. Note that the integer $n^{*}$ is finite since $Q_{i j}^{\#(n)}(z, y, t) \underset{n \rightarrow \infty}{\longrightarrow} 0$ for a normal MRP with fixed values of $i, j \in E, t>0, z>0$ and $y \in[z, \Delta]$.

\subsection{Numerical results}

To check the validity of our results, let us study the process $Z_{t}$ governed by

$$
\dot{Z}_{t}=a Z_{t} \times X_{t}, \quad Z_{0}=z
$$


with $a=0.0075, z=10, \Delta=50$. The process $X_{t}$ is a three-state jump Markov process with $E=\{1,2,3\}$, a matrix generator and an initial law given by

$$
\mathbf{A}=\left[\begin{array}{ccc}
-0.02 & 0.02 & 0 \\
0.027 & -0.03 & 0.003 \\
0.01 & 0 & -0.01
\end{array}\right], \quad \alpha=\left[\begin{array}{lll}
\frac{2}{3} & \frac{1}{3} & 0
\end{array}\right] \text {. }
$$

The coupled process $\left(Z_{t}, X_{t}\right)$ defines a PDMP whose transition function and reliability function can be computed through Proposition 2 and (11), based upon the numerical implementation described above for which we put $M=L=100$ points of discretization. Another way to compute the reliability is the Monte-Carlo method, which consists in simulating a large number of paths of $Z_{t}$ and counting when the state $\{\Delta\}$ is reached or not. We use the empirical estimator applied on $K=10000$ paths $\left(Z_{t}^{k}\right)_{k=1, \ldots, K}$ simulated through Monte-Carlo techniques, that is $\widehat{R}(t)=\frac{1}{K} \sum_{k=1}^{K} \mathbb{1}_{\left\{Z_{t}^{k}<\Delta\right\}}$. This estimator is compared with the direct calculus of $R$ through the Markov renewal argument developed in this paper. Results can be found on Figure 2 where the Monte-Carlo estimator is used as a reference to check the validity both of the theoretical results and the numerical implementation. One can see that the reliability curves obtained with the two methods are quite close.

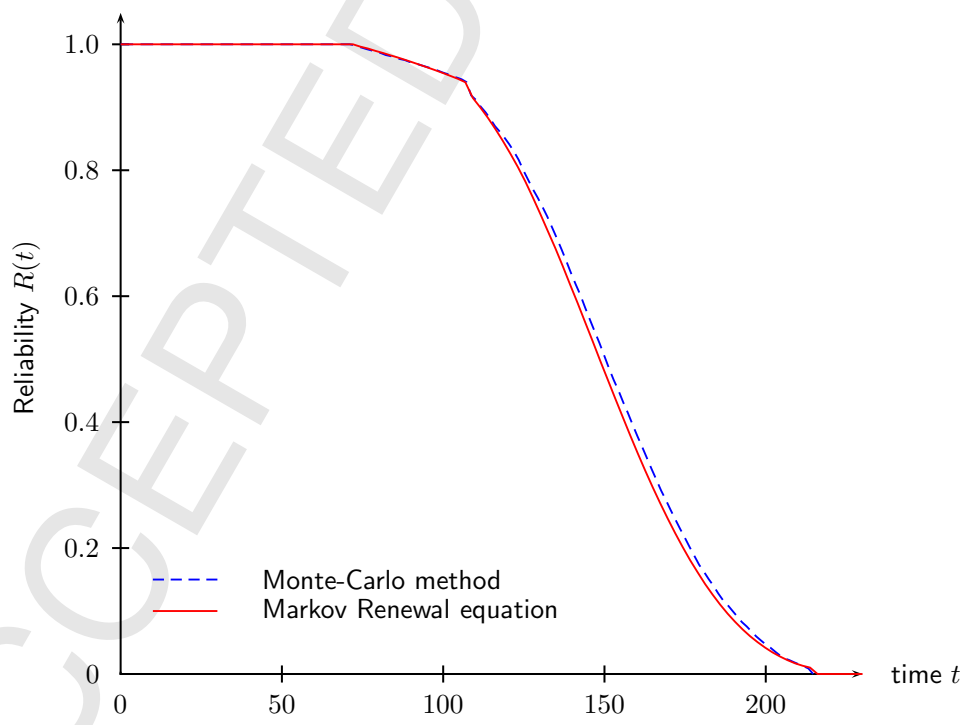

Fig. 2. Reliability estimation with two different methods 


\section{Concluding remarks}

Because models based on system (1) are encountered in the literature for many different fields of applications, we think that the reliability results of this paper may be useful for engineering studies, such as structural mechanics (see, e.g., Chiquet et al., to appear). Yet, to be more suitable to real phenomena, the case of PDMP which are not only monotone should be considered, as further developments.

\section{References}

Chiquet, J., Limnios, N., 2006. Estimating stochastic dynamical systems driven by a continuous-time jump markov process. Methodology and Computing in Applied Probability 8, 431-447.

Chiquet, J., Limnios, N., Eid, M., to appear. Mathematical methods in survival analysis, reliability and quality of life. Hermès, Ch. Reliability of stochastic dynamical systems applied to fatigue crack growth modelling.

Davis, M., 1984. Piecewise-deterministic Markov processes: a general class of nondiffusion stochastic models. Journal of the Royal Statistical Society 46, 353-388.

Davis, M., 1993. Markov models and Optimization. Monographs On statistics and Applied Probability 49. Chapman \& Hall.

Devooght, J., Smidts, C., 1996. Probabilistic dynamics as a tool for dynamic psa. Reliability Engineering and System Safety 52, 185-196.

Embrechts, P., Frey, R., Furrer, H., 2001. Stochastic Processes: Theory and Methods. Vol. 19 of Handbook of statistics. Elsevier Science, Amsterdam, Ch. Stochastic Processes in Insurance and Finance, pp. 365-412.

Jacobsen, M., 2006. Point Process Theory and Applications. Birkhäuser.

Koroliuk, V., Limnios, N., 2005. Stochastic Systems in Merging Phase Space. World Scientific.

Lapeyre, B., Pardoux, E., 2003. Introduction to Monte-Carlo Methods for Transport and Diffusion Equations. Oxford University Press.

Limnios, N., Opriçan, G., 2001. Semi-Markov processes and Reliability. Birklhaser. Øksendal, B., 2003. Stochastic differential equations, sixth Edition. Springer. 